
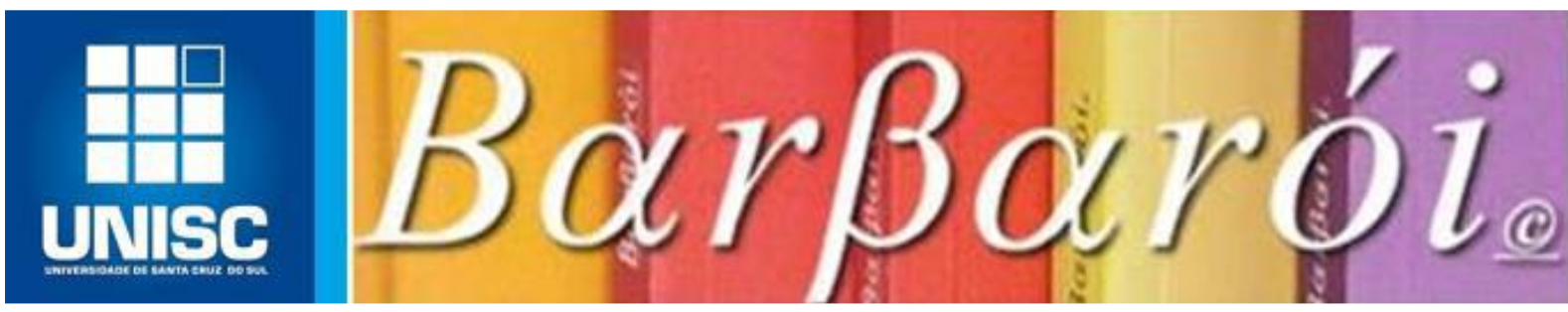

\title{
RECOMPOSICIÓN NEOLIBERAL Y REFORMAS LABORALES EN EL MERCOSUR: LOS CASOS DE ARGENTINA Y BRASIL (2015-2020)
}

DOI: http://dx.doi.org/10.17058/barbaroi.v0i57.15061

\author{
Alejandro Frenkel \\ Universidad Nacional de San Martín - UNSAM - Argentina \\ Bruno Dobrusin \\ Universidad de Buenos Aires - UBA - Argentina \\ $*$
}

\section{Resumen}

El artículo analiza los cambios en la agenda laboral de los países del Mercosur y su vinculación con el perfil que asumió el bloque tras la llegada de gobiernos de corte neoliberal a mediados de la década de 2010. En este marco, se presta especial atención a las iniciativas de reforma del mercado de trabajo impulsadas por los gobiernos de Michel Temer y Jair Bolsonaro en Brasil y Mauricio Macri en Argentina. Reformas que buscaron favorecer la "competitividad" de las economías del bloque, apostando por una integración periférica en las cadenas globales de valor.

Palabras clave: Mercosur; neoliberalismo; agenda laboral; Argentina; Brasil

\section{Introducción}

La agenda laboral se ha transformado en un tema central de las discusiones globales y regionales en materia de políticas estatales. Mientras crece el debate sobre el futuro de las relaciones laborales en función de la robotización y la automatización de las condiciones de producción (FREY y OSBORNE, 2017; GRAETZ y MICHAELS, 2018; LARIVIÈRE ET AL. 2017; ACEMOGLU y RESTREPO, 2019; SCHWAB, 2016; SOTO, 2020; MAKRIDAKIS, 2017), en el ámbito regional viene ganando lugar la cuestión de cómo adaptar las economías latinoamericanas a un escenario internacional que se conjuga sobre un parte aguas: por un lado, en Occidente se está dando un creciente cuestionamiento a la globalización neoliberal. Entre otras 
cosas, esta impugnación se sustenta en una retórica de defensa de los entramados productivos nacionales ${ }^{1}$. Como otra cara de la misma moneda, el ascenso de China y los países de Asia-Pacífico como centro de gravitación mundial han traído una novedad: son estos países quienes reivindican la globalización y las agendas de liberalización comercial. En este marco, las políticas de desregulación y apertura económica no están desapareciendo sino que hay un cambio de mando en sus principales promotores. En el medio de estos dos polos juegan, desde luego, las corporaciones trasnacionales.

Resulta inevitable que este escenario impacte en América Latina. Más aún, si se tienen en cuenta los movimientos que se dieron en la región con la llegada de gobiernos neoliberales en países como Argentina y Brasil entre 2015 y 2016. Este cambio trajo aparejado, entre otras cosas, una proclama de mayor apertura económica, motorizando las economías en función de las inversiones externas y una profundización del perfil de países exportadores de commodities (ZELICOVICH, 2018; BERRINGER y FORLINI, 2018; MOREIRA, 2020). Esta concepción de un modelo de desarrollo que se articula de afuera hacia adentro favorece un escenario de mayor vulnerabilidad frente a las presiones de los actores económicos y financieros para desregular el mercado de trabajo. El argumento, en este caso, radica en aumentar la competitividad, bajar los costos de producción y proveer una inserción “eficiente” en las cadenas globales de valor.

La adopción de este esquema de inserción internacional también tiene sus implicancias en los esquemas latinoamericanos de integración: las visiones comercialistas recuperaron terreno, reeditando una especie de regionalismo abierto recargado (QUILICONI, 2018), impulsando iniciativas para flexibilizar los organismos de integración. En el caso del Mercosur, el objeto de ello sería mitigar, o directamente eliminar, las "rigideces" institucionales, normativas y políticas que pudieran condicionar una inserción competitiva en los mercados globales, aun cuando ello implicara suprimir o desconocer las normas y los mecanismos que promueven la negociación en bloque (COMINI y FRENKEL, 2017). En este contexto, Mercosur se transformaría en una herramienta comercial para competir globalmente, más que como un proyecto de integración autonómico y multidimensional.

Dicho esto, este trabajo da cuenta de los cambios en la agenda laboral de los países del Mercosur y su vinculación con el nuevo perfil que asumió el bloque tras la llegada de gobiernos

\footnotetext{
${ }^{1}$ El Brexit, el "America First" de Donald Trump, las proclamas xenófobas en algunos países europeos o la cruzada contra el "dumping social" que está encarando el presidente francés, Emmanuel Macron, son algunos ejemplos de ello.
} Barbarói, Santa Cruz do Sul, n. 57, p.<08-28>, jul./dez. 2020 
neoliberales de Mauricio Macri en Argentina y Michel Temer en Brasil, en 2015 y 2016 respectivamente. En este marco, se presta especial atención los planes de reforma laboral que fueron implementados y discutidos en estos países en pos de favorecer la inserción internacional y “competitividad” de las economías de la región.

La investigación parte de una metodología cualitativa, de tipo flexible, correspondiéndose con lo que Stephen Van Evera denomina como investigación "histórica-explicativa". Es decir, un estudio orientado a explicar causas, patrones y consecuencias de casos históricos (VAN EVERA, 1997). En base a ello, el artículo se divide en tres partes: en la primera se realiza un recorrido histórico de los diferentes perfiles que fue teniendo el Mercosur, puntualizando en las áreas y agendas que tuvieron mayor preeminencia y en las visiones sobre la inserción internacional del bloque. La segunda parte vincula lo anterior con el desarrollo de la agenda laboral, desde la etapa de formación del Mercosur hasta la llegada de gobiernos de corte neoliberal a mediados de la década de 2010. Por último, en la tercera sección se analizan las iniciativas de reformas del mercado de trabajo impulsadas por los gobiernos de Michel Temer y Jair Bolsonaro en Brasil y Mauricio Macri en Argentina y su relación con el perfil que asumiendo el Mercosur en esta etapa.

\section{Del Mercosur social al Mercosur comercialista}

Todo proceso de integración es un proceso histórico en constante construcción. El bloque conosureño nacido en 1991 es, tal vez, uno de los proyectos del regionalismo latinoamericano que mayores transformaciones sufrió en las últimas décadas. Siguiendo a Mercedes Botto (2011), hasta hace algunos años se podían identificar al menos tres etapas en el desarrollo del Mercosur. En la primera (1987-1991), los gobiernos de Argentina y Brasil buscaron alcanzar una integración económica bajo un esquema de especialización intraindustrial, de carácter gradual sectorial y flexible, mediante protocolos sectoriales. La segunda etapa (1991-2002) estuvo definida por la incorporación de Paraguay y Uruguay y por el predominio de un fuerte sesgo comercialista, en el marco del cual los mercados marcaron el ritmo y el carácter de la integración ${ }^{2}$. La siguiente etapa se abriría luego de la crisis de 2001 y su característica central es el retorno del liderazgo estatal, el proceso de incorporación de Venezuela y el consenso sobre la necesidad de producir un vuelco

\footnotetext{
${ }^{2}$ Cabe destacar que este sesgo comercialista no impidió que se incorporaran otras áreas al proceso de integración. Por caso, para mediados de la década ya se habían institucionalizado mecanismos de cooperación en temas como Justicia, Agricultura, Medio Ambiente, Educación y, como se verá más adelante, también en el área de Trabajo.
}

Barbarói, Santa Cruz do Sul, n. 57, p.<08-28>, jul./dez. 2020 
hacia un Mercosur más inclusivo. Tras el retorno de Tabaré Vázquez a la presidencia de Uruguay, los cambios de gobierno que se sucedieron entre 2015 y 2016 en Argentina y Brasil y la profundación de la crisis venezolana, el Mercosur entró en una nueva fase.

Si bien en cada una de estas etapas se pueden encontrar cambios y continuidades; reediciones y refundaciones, a los efectos de este trabajo nos interesa hacer foco en las características centrales de la etapa que se abrió en los albores del siglo XXI y aquella que se inicia, entre 2015 y 2016, con el arribo de gobiernos neoliberales en Argentina y Brasil.

Luego de que las reformas estructurales y desregulación de la economía que caracterizaron a la década de 1990 perdieran legitimidad, los procesos de integración regional empezaron a transformarse. La vinculación entre "integración" y "mercado" dio paso a un modelo que reincorporaba las dimensiones políticas y sociales de la integración. En este marco, la política de integración regional de la mayoría de los países del Cono Sur se circunscribiría en un momento de expansión de gobiernos de centro izquierda que proponían cambios en el modelo de desarrollo hacia parámetros que podrían denominarse como "neodesarrollistas" (BRESSER- PEREIRA, 2007, 2016; FÉLIZ, 2012; BRESSER-PEREIRA, OREIRO y MARCONI, 2014).

Para muchos gobiernos latinoamericanos, los proyectos de regionalismo adquirieron por esos años un carácter de resistencia (HETTNE, 2003) frente a un proceso de globalización percibido como irreversible, pero con efectos que acentúan el carácter desigual del desarrollo y con peligrosas consecuencias sociales (FERNÁNDEZ, 2004: 21). En este marco, la integración regional fue relanzada bajo un prisma posliberal (SANAHUJA, 2009); inclusivo (VÁZQUEZ, 2011); post-hegemónico (RIGGIROZZI y TUSSIE, 2012) o estratégico (BRICEÑO RUIZ, 2008; BIZZOZERO, 2011) y paulatinamente se fueron ampliando las agendas más allá de la económicocomercial incorporando, en cambio, un carácter multidimensional (SERBIN, 2011; ROJAS ARAVENA, 2012; RIGGIROZZI y GRUGEL, 2015). Esta idea multidimensional e integral que comenzó a permear a los procesos de integración a comienzos del siglo XXI tuvo, en el Mercosur, una serie de indicadores concretos. El primer hito fue el impulso del "Mercosur Social", mediante la firma de la Decisión No 61/00 del Consejo del Mercado Común (CMC). Decisión que instauraba la Reunión de Ministros y Autoridades de Desarrollo Social del bloque. A partir de allí comenzaría el tránsito hacia una nueva definición de lo social y hacia un nuevo espacio de esta dimensión en el proceso de integración (PERROTTA y VAZQUEZ, 2010). 
El segundo hito del proceso mercosureño por aquellos años fue el establecimiento de los que se conoció como el Consenso de Buenos Aires (2003) entre Lula da Silva y Néstor Kirchner³. Este marco de transformación y relanzamiento de la alianza estratégica sirvió para dar un nuevo impulso regional (BIZZOZERO, 2011). La centralidad de la agenda económica comenzó a desdibujarse y, en cambio, cobró fuerza la idea de un proceso articulado alrededor de múltiples dimensiones. Además de los asuntos sociales, laborales, ambientales, de seguridad o de salud, en esta etapa del Mercosur también se buscó reposicionar a la variable política como el motor del proceso integracionista. Con esta idea se inauguró la Comisión de Representantes Permanentes del Mercosur (que más tarde sería reemplazada por el Alto Representante del Mercosur) y se llamaría a iniciar un proceso de reforma institucional, con el objetivo de adaptar la arquitectura mercosureña a los nuevos tiempos. Tanto la ampliación de las agendas sectoriales como la reconfiguración de la dimensión política fueron visualizados por los gobiernos neodesarrollistas como herramientas para desandar el "Mercosur fenicio" (CAETANO, 2006) que había tenido su esplendor durante la década de 1990. Es decir, un bloque con muy escasa institucionalidad y acaparado por la agenda económico-comercial.

Ahora bien, más allá de las intenciones, los resultados de la reconfiguración institucional del Mercosur fueron, más bien, ambivalentes. El aspecto político en términos de alcanzar una mayor capacidad de decisión autónoma encontró un lugar destacado a la hora de aunar posiciones frente a determinados acontecimientos regionales. La articulación de una postura común durante la Cumbre de las Américas de 2005 que echó por tierra la aprobación del Área de Libre Comercio para las Américas (ALCA) o la suspensión de Paraguay luego de la fraudulenta destitución de Fernando Lugo en 2012 son algunos de los ejemplos más destacables. No obstante, este accionar sería más el resultado de una activa "diplomacia presidencial” que de la utilización efectiva de los mecanismos políticos existentes, como el Foro de Consulta y Concertación Política. Otro tema que reveló las dificultades y divergencias internas en el bloque fue la negociación conjunta frente a actores extrarregionales. Por caso, ante la creciente presencia de China en la región los países del

\footnotetext{
${ }^{3}$ El Consenso de Buenos Aires fue un documento suscripto por los entonces presidentes de Argentina y Brasil el 16 de octubre de 2003. Entre otras cosas, allí se afirmaba la necesidad de encarar la integración sobre la base de una distribución equitativa de la riqueza y la inclusión social; una mayor participación del Estado y la búsqueda de mayores márgenes de autonomía que, asimismo, permitan consensuar posiciones comunes para negociar con terceros. Sumado a ello, se afirmaba que el trabajo decente, tal como es concebido por la OIT, es el instrumento más efectivo de promoción de las condiciones de vida de nuestros pueblos. El Consenso de Buenos Aires se plasmó luego en el Programa de Trabajo 2004-2006 (adoptado por la decisión CMC N ${ }^{\circ} 26 / 03$ ).
}

Barbarói, Santa Cruz do Sul, n. 57, p.<08-28>,jul./dez. 2020 
bloque terminaron adoptando un relacionamiento bilateral, aun cuando las agendas de los Estados miembros con el gigante asiático fueran similares. La negociación del tratado comercial con la Unión Europea es otro ejemplo de cómo se fue licuando la dimensión política del bloque: ante las dificultades para alcanzar una posición consensuada, los gobiernos de Brasil, Paraguay y Uruguay comenzaron a ceder a las presiones internas para avanzar en una negociación a varias velocidades con el bloque europeo, lo cual contrastaba con la posición argentina, mucho más proteccionista y reacia a la negociación (SANAHUJA y RODRÍGUEZ, 2019).

La reforma institucional, iniciada en 2005 a través de la conformación del Grupo de Alto Nivel para la Reforma Institucional (GANRI) con el objeto de reducir la impronta económica del Mercosur, quedaría trunca por la oposición, principalmente, de las burocracias estatales nacionales. Esto significó que aun en un proceso de redefinición del regionalismo conosureño las viejas estructuras debieran convivir con las nuevas (INCHAUSPE y PEROTTA, 2008), complejizando la puesta en marcha de agendas no comerciales.

Entre 2015 y 2016 se sucedieron una serie de hechos políticos en América Latina que abrieron el debate respecto de si la región se encontraba en una especie de cambio de ciclo, marcando un punto de inflexión con la etapa de gobiernos de centro izquierda ${ }^{4}$. En este convulsionado contexto, los procesos de integración entraron en una nueva etapa de redefiniciones. La Unión de Naciones Suramericanas (UNASUR) fue vaciada y reemplazada por el Foro para el Progreso de América del Sur (PROSUR), un organismo a medida de los gobiernos conservadores de turno (HERRERA, 2019). En el caso del bloque mercosureño, si en la etapa anterior se había intentado configurar -en mayor o menor medida- como una herramienta de autonomía y contrabalance de los efectos asimétricos de la globalización, con la llegada de los nuevos gobiernos liberales se dio un reposicionamiento del regionalismo abierto y los procesos de integración pasaron a ser concebidos como un instrumento para atraer capitales e "insertarse" en los mercados globales.

\footnotetext{
${ }^{4}$ La derrota de Evo Morales en el referendo del 21 febrero de 2016, el fracaso del oficialismo venezolano en las elecciones legislativas de diciembre de 2015, la victoria de Mauricio Macri en Argentina, también en 2015, y el impeachment a Dilma Rousseff en Brasil fueron los indicadores más relevantes de inicio de cambio de tendencia en la región, que luego se profundizaría con el viraje ideológico del gobierno de Lenín Moreno en Ecuador, la llegada de Jair Bolsonaro a la presidencia brasileña, el golpe de Estado contra Morales en Bolivia y la victoria de Luis Lacalle Pou en Uruguay.
} 
A raíz de ello, los gobiernos de derecha de la región redoblaron las pulsiones para flexibilizar el perfil del Mercosur y lograr una mayor convergencia con la Alianza del Pacífico ${ }^{5}$. La apelación a la flexibilidad implicaría reintroducir el cortoplacismo de las "alianzas ad-hoc", estableciendo un Mercosur "sin ataduras" para negociar individualmente (CABALLERO, 2019). En la práctica, flexibilizar el bloque significa deshacer toda rigidez institucional, normativa y política que pudiera impedir o, cuanto menos, condicionar una inserción competitiva en los mercados globales. En un mismo sentido, el resultado de este movimiento es un indicador de utilidad para los sectores que revalorizan las zonas de libre comercio en detrimento de los proyectos con perspectivas más ambiciosas, como puede ser la conformación de un mercado común o priorizar la generación de políticas públicas regionales en otras agendas más allá de las económicocomerciales (COMINI y FRENKEL, 2017).

En definitiva, en la etapa de regionalismo abierto recargado, las agendas comerciales volvieron a ganar terreno como el lei motiv del Mercosur, erosionando la idea de multidimensionalidad y, con ella, la posibilidad de potenciar la cooperación en las agendas sociales, políticas y, también, laborales. A menos, claro, que las mismas se articulen como un apéndice de un modelo de inserción periférica en las cadenas globales de valor.

\section{Avances y contradicciones de la agenda laboral del Mercosur}

Dentro de las diferentes agendas que convergen en el Mercosur, la agenda laboral es una de las pocas donde la participación de actores externos a los gobiernos tiene mayor relevancia. Incluso desde antes de la fundación del Mercosur, el sindicalismo ha sido un actor central en la discusión, presión e implementación de los temas laborales en el proceso de integración sudamericano. En este sentido, los sindicatos de la región "corren con ventaja" frente a los gobiernos y otros actores de la sociedad civil, ya que desde la salida de las dictaduras la integración regional era percibida por éstos como una herramienta estratégica (BOTTO, 2015). En 1986, se crea la Coordinadora de Centrales Sindicales del Cono Sur (CCSCS), integrada por centrales

\footnotetext{
${ }^{5}$ Paraguay es miembro observador de la AP desde el año 2013. En 2016, Argentina se incorporó bajo la misma modalidad. Uruguay, por su parte, formalizó su pedido de entrar al bloque del Pacífico como "Estado asociado". Cabe mencionar que hasta el momento, la Alianza del Pacífico no ha configurado un bloque institucionalizado en una estructura concreta. Su objetivo está centrado en la concreción de acuerdos de libre comercio entre sus miembros, similar al esquema establecido en el Tratado de Libre Comercio de América del Norte (TLCAN).
}

Barbarói, Santa Cruz do Sul, n. 57, p.<08-28>, jul./dez. 2020 
sindicales de Argentina, Uruguay, Paraguay, Brasil, Bolivia y Chile ${ }^{6}$. En su origen, la CCSCS tenía como objetivo fortalecer, desde el accionar sindical, los procesos de democratización y refuerzo de derechos sociales que estaban experimentando los países de la región (CARRAU, 2008).

Desde sus comienzos, el bloque sindical actuó a través de la CCSCS para influenciar a los gobiernos, promoviendo la conformación de una alianza estratégica entre Argentina y Brasil. Esto significó que en el nacimiento y posterior desarrollo del Mercosur el sector obrero tuviera la capacidad de incidir en la agenda laboral, a partir de una trayectoria previa de colaboración y preparación. Asimismo, la incidencia sindical permitió que el Mercosur combinara aspectos puramente "libre-cambistas", típicos de la etapa neoliberal, con instituciones de diálogo social inspiradas en aquellas creadas al interior de la Unión Europea. Como resultado de esta particular combinación, durante la etapa más regresiva en términos de reformas laborales en los países del Mercosur igual se daría un sustantivo avance de la institucionalidad laboral al interior del bloque (ROBLES, 2002). Incluso mayor que en otras épocas de mejoras en indicadores sociales y económicos, como fueron los años de gobiernos neodesarrollistas (DOBRUSIN, 2012).

En efecto, durante los primeros diez años del Mercosur se crearon instituciones vinculadas a la agenda laboral que se mantienen hasta la actualidad. Entre las tres principales están: el Comité Socio-Laboral del Mercosur, el Subgrupo de Trabajo 10 (sobre acciones de carácter laboral) y el Foro Consultivo Económico y Social (FCES). Estos espacios fueron creados en la segunda mitad de los noventa (en gran parte, por la presión sindical) y tienen un carácter tripartito en la deliberación. En términos de decisiones finales, el Subgrupo de Trabajo 10, que influye sobre las reglas a implementar por el bloque, continúa estando en manos de los gobiernos. El FCES, por su parte, reúne a organizaciones de la sociedad civil e incluye a sindicatos y empresarios. Su influencia es baja debido a que sus decisiones tienen que ser negociadas por país, con cada una de las partes participantes y luego consensuadas a nivel regional. Además, las decisiones tienen un carácter, como lo indica su título, consultivo, con lo cual los gobiernos y las autoridades del Mercosur no están obligadas a adoptarlas.

\footnotetext{
${ }^{6}$ La CCSCS fue mutando su composición con el transcurrir de los años. Si bien en el origen incorporó centrales sindicales de países externos a lo que luego sería el Mercosur, el núcleo central de actuación son las organizaciones de los 4 países firmantes del Tratado de Asunción; Argentina, Uruguay, Paraguay y Brasil. Desde el ingreso de Venezuela, sindicatos de ese país también comenzaron a participar de esta coordinadora.
} 
La etapa de crecimiento de la institucionalidad laboral del Mercosur (1995-1999) coincide con momentos de crisis económicas al en los miembros del bloque que afectaron la situación del mercado laboral. Sobre todo, el crecimiento del desempleo, la caída de los salarios y la profundización de la flexibilización laboral (COOK, 2007). Esto explica, a nuestro entender, el mayor accionar sindical en la agenda regional: al cerrarse posibilidades y espacios de negociación a nivel nacional, las centrales sindicales buscaron orientar la presión hacia el ámbito regional buscando obtener herramientas que les permitieran conservar los derechos adquiridos. La Declaración Sociolaboral del Mercosur, concertada en 1998, es un ejemplo de este accionar. En línea con la declaración de principios fundamentales de la Organización Internacional del Trabajo (OIT), la declaración establece derechos individuales (no discriminación en el trabajo, eliminación del trabajo forzoso, derecho a migrar) y colectivos (derecho a huelga, a la organización y negociación colectiva) que deben ser respetados y promovidos por todos los países miembros. Además, la declaración estableció una serie de metas para el bloque que superan los aspectos estrictamente comerciales, al incluir la necesidad de promover el empleo, garantizar los mecanismos de seguridad social y promover el desarrollo social (MERCADO COMÚN DEL SUR, 1998). Si bien esto puede sonar meramente declarativo, la declaración puede leerse como un triunfo en la agenda sindical, en tanto ponía un freno a la idea predominante en algunos gobiernos de la época de que el Mercosur debía parecerse más al Tratado de Libre Comercio de América del Norte (TLCAN). Es decir, un esquema con la menor institucionalidad posible y un énfasis exclusivo en el comercio.

Ahora bien, más allá de los avances institucionales del Mercosur alcanzados durante la década del noventa, las instituciones laborales del bloque poseen una serie de características que merecen ser remarcadas: alta informalidad; baja participación por parte del empresariado; intensa participación de los sindicatos y el carácter "consultivo" de sus decisiones son las más predominantes (ROBLES, 2006, p. 72-73). Estas características indican que los avances en la temática laboral están fuertemente relacionados con la capacidad sindical de influenciar a los gobiernos de manera directa. A su vez, indican la escasa predisposición de los gobiernos nacionales y el sector empresarial para establecer instituciones laborales con participación integral y capacidad de decisión vinculante. Por último, cabe destacar que la capacidad de incidencia sobre ministerios como los de trabajo y desarrollo social suele ser mayor para los actores sindicales, mientras que las carteras de economía, infraestructura y las cancillerías tienen menor predisposición a la Barbarói, Santa Cruz do Sul, n. 57, p.<08-28>, jul./dez. 2020 
participación. Con el agravante de que son estas últimas las que suelen llevar adelante las principales decisiones sobre los procesos de integración.

De igual forma, vale remarcar que estas características se han mantenido prácticamente inmutables hasta la actualidad. En efecto, aun cuando proliferaron los gobiernos de centroizquierda y hubo mayor interés por los temas laborales, la densidad de las acciones se basó en la institucionalidad creada durante los años noventa. En este sentido, existen más líneas de continuidad que de ruptura entre el Mercosur neoliberal de los noventa y la etapa del Mercosur social de la década de 2000. Como se dijo anteriormente, los gobiernos de centroizquierda concibieron a la integración regional como una herramienta para obtener mayores márgenes de autonomía. Y si bien durante esta etapa el Mercosur se potenció a nivel político-estratégico, no se logró profundizar sobre medidas específicas que permitieran romper con el molde original del bloque (DOBRUSIN, 2012). Los avances en la agenda laboral durante la etapa progresista dependieron, sobre todo, de la capacidad de los sindicatos de influenciar directamente a los presidentes. Las medidas respecto a los avances en los derechos migratorios dentro del bloque, así como la creación del Instituto Social del Mercosur y el Instituto de Derechos Humanos fueron, en gran parte, producto del accionar presidencial. Esto refuerza la idea presentada por Malamud (2005) sobre el carácter "interpresidencialista" del Mercosur, en el que la institucionalidad tiene un segundo plano y el accionar de los Jefes de Estado es determinante en el devenir del bloque.

Tomando en cuenta la característica mencionada en el párrafo anterior, uno de los ejes de presión durante los gobiernos progresistas fueron las cumbres sociales del Mercosur (BOTTO, 2015). Si bien éstas ya existían en los años noventa, tomaron mayor notoriedad durante la etapa del Mercosur social, inclusive contando en diversas ocasiones con la participación de figuras importantes de los gobiernos y hasta de los propios presidentes. Estas cumbres permitieron escenificar la idea de un Mercosur contrapuesto al del neoliberalismo, orientado en términos retóricos hacia la integración productiva y con mayor participación social. Sin embargo, durante esta etapa las mejoras en los indicadores nacionales en términos laborales (caída del desempleo, incremento de los empleos formales, crecimiento del salario real impulsado por el salario mínimo) no tuvieron una correlación en el nivel regional. Con la excepción de los nuevos derechos migratorios (DOBRUSIN, 2012), la agenda laboral de la etapa progresista tuvo similitudes y ritmos similares a los de la etapa neoliberal. El hito más relevante en cuestiones laborales fue la revisión de la Declaración Sociolaboral del Mercosur, realizada en 2015, en la que se avanzó en una mayor 
protección de los derechos laborales y sindicales, se explicitó el derecho a huelga, se incorporó la protección ante el despido y se incluyó una misma jornada laboral de ocho horas para todos los países miembros. La declaración también incorporó agendas laborales internacionales que se discutieron desde 1998, en especial en lo relativo al Trabajo Decente y a los derechos de los Trabajadores Migrantes (MERCADO COMÚN DEL SUR, 2015).

La baja densidad de medidas concretas en las instituciones laborales del Mercosur también estuvo relacionada a la presencia sindical en las alianzas gubernamentales de la mayoría de los países del bloque (Argentina, Brasil y Uruguay). Esto implicó un mayor enfoque en generar influencia mediante los canales de participación y negociación abiertos en la esfera nacional, en lugar de buscar la institucionalidad supranacional como mecanismo de influencia (BOTTO, 2015).

\section{Recomposición neoliberal y reformas laborales en Argentina y Brasil}

Si bien durante la etapa del Mercosur social la agenda laboral no avanzó a la par de la retórica integracionista, lo cierto es que la ampliación de las discusiones regionales y, sobre todo, el carácter de las políticas nacionales terminó beneficiando indirectamente el avance en materia de derechos. Los sindicatos, por su parte, se fortalecieron a la par de las mejoras en los indicadores de mercado de trabajo (COOK, 2011). De igual forma, algunas de las decisiones geopolíticas, como el ingreso de Venezuela como miembro pleno, no tuvieron repercusión directa en la cuestión laboral, pero sí implicaron una profundización de la integración al ampliar el espacio geográfico (sudamericano) y sectorial (energético).

Por otro lado, la impronta que adquirió el Mercosur tras el giro liberal-conservador impulsado por Macri en Argentina y Temer en Brasil puso en cuestión la idea de una integración más profunda. Como se dijo anteriormente, un denominador común de estos gobiernos fue el de impulsar un perfil más comercialista para el organismo, propiciando políticas regionales que sirvan para atraer inversiones externas y quitar las protecciones a los sectores industriales. Estos factores, suplementados por la crisis política y económica en Venezuela, se conjugaron para redireccionar el Mercosur hacia un esquema que, en cierta forma, reeditaba el regionalismo abierto. En este marco se desarrollaron tres agendas que afectarían la agenda laboral del bloque y que directa e indirectamente impactarían en las realidades de los trabajadores en todos los países miembros: las reformas laborales; el acuerdo de libre comercio con la Unión Europea y la profundización de las relaciones con Estados Unidos. De estas tres cuestiones, sólo el acuerdo comercial con el viejo 
continente fue negociado en bloque ${ }^{7}$. Las otras dos, si bien afectan seriamente al proceso mercosureño, avanzaron por carriles nacionales independientes del resto de los miembros.

El primero de los países del bloque que avanzó con la agenda de reformas laborales fue Brasil. En el marco de un programa político y económico orientado a redefinir las relaciones entre Estado, mercado y sociedad civil, el gobierno de Michel Temer impulsó a mediados de 2016 una reforma integral de los derechos laborales y sindicales de los trabajadores brasileños. Entre sus consecuencias más importantes, la reforma flexibiliza las formas de contratación, permite que las negociaciones colectivas tengan un grado superior a la ley, avanza sobre derechos sindicales, permitiendo extender la jornada de trabajo (TEIXEIRA, 2017) ${ }^{8}$. La agenda de reforma laboral se siguió profundizando tras la llegada de Jair Bolsonaro a la presidencia, en enero de 2019. Promovidas por el superministro de economía y alumno de la Escuela de Chicago, Paulo Guedes, el nuevo gobierno colocó el tema dentro de un paquete más amplio de reformas estructurales, orientadas a "aumentar la competitividad y desburocratizar la economía" (IRAJÁ, 2019). Dicho paquete incluía también una reforma previsional, medidas de reducción del gasto público, un ambicioso programa de privatizaciones y un proyecto de reforma administrativa que procuraba modificar las reglas del empleo público a través del congelamiento de concursos públicos, flexibilización de las jornadas de trabajo y reducción de los salarios (PASSARINHO, 2019). Asimismo, el gobierno bolsonarista decidió la eliminación del ministerio de trabajo y sus funciones pasaron a distribuirse en las carteras de economía, de ciudadanía y de justicia. Sin embargo, aun cuando se logró avanzar en algunos de estos puntos, el clima de protestas que estalló en la región a fines de 2019 -y que se dio principalmente en países con gobiernos de derecha, como Chile o Colombia- llevaron a Bolsonaro y a Guedes a frenar la reforma laboral, posponiendo el proyecto de reforma administrativa que procuraba modificar las reglas del empleo público ${ }^{9}$.

Casi al mismo tiempo que Temer en Brasil, en Argentina también se desarrolló un clima favorable a las reformas en el mercado de trabajo. Con el objetivo de "bajar el costo laboral" (PAGNI, 2017; ZULETA, 2017; BONELLI, 2017) y potenciar una inserción "eficiente" en las cadenas globales de valor, el gobierno de Macri puso en agenda una reforma similar a la del país

\footnotetext{
${ }^{7}$ En junio de 2019, luego de largo proceso de negociaciones, la Unión Europea y Mercosur anunciaron la conclusión del capítulo comercial de su Acuerdo de Asociación.

${ }^{8}$ Para ver un análisis pormenorizado de la reforma laboral se recomienda el trabajo realizado por el CESIT (2017).

${ }^{9} \mathrm{Al}$ respecto, el propio Guedes expresó que "cualquier país democrático, cuando ve al pueblo saliendo a las calles, se pregunta si vale la pena hacer tantas reformas al mismo tiempo" (BAE NEGOCIOS, 2019).
}

Barbarói, Santa Cruz do Sul, n. 57, p.<08-28>,jul./dez. 2020 
verde amarelo, que incluía también una reforma de la seguridad social, aumentando la edad jubilatoria y los años de contribución efectiva. No obstante, los vientos de reforma chocarían con la resistencia del movimiento sindical y con un humor social crispado, producto de la crisis económica iniciada en abril 2018 luego de una fuerte devaluación del peso. Ahora bien, aun cuando la reforma no pudo ser implementada en los términos planteados inicialmente, sí se logró avanzar en un esquema de reformas parciales y graduales, a partir de negociaciones colectivas por sector. El convenio laboral acordado en 2017 entre el gobierno nacional, la cámara empresarial y el sindicato petrolero para la explotación hidrocarburífera no convencional en el yacimiento "Vaca Muerta" se volvería un emblema de este tipo de reformas parciales. Entre sus puntos más destacados, dicho convenio permite la tercerización y flexibilización en la contratación de trabajadores, extiende la jornada laboral, reduce el tiempo de descanso de los trabajadores y elimina las horas extras. A ello se sumó el impulso ese mismo año de "nuevo convenio automotriz", que buscaría replicar el convenio de Vaca Muerta al sector metalmecánico. El acuerdo, entre otras cosas, habilita a las empresas a rotar el personal, prolongar los contratos de trabajo, reducir las jornadas de descanso y establecer un sistema de suspensiones rotativas "justificadas por restructuración de las plantas" (BARRAZA, 2017). Al respecto, cabe destacar que, aunque con diferencias, este mecanismo de reformas por sectores específicos sería avalado por el gobierno peronista de Alberto Fernández, quien asumió la presidencia en diciembre de 2019 tras derrotar a Mauricio Macri en las elecciones generales ${ }^{10}$.

Como se dijo anteriormente, el acuerdo de libre comercio con la Unión Europea fue uno de los puntos en los que el Mercosur pudo mantener una negociación conjunta, bloque a bloque. Respecto del impacto en el mercado laboral, vale resaltar que, de avanzar en los términos acordados en junio de 2019, la implementación afectaría negativamente a los sectores industriales argentinos y brasileños, ya que la competencia con el empresariado europeo sería asimétrica sin las protecciones aduaneras que rigen actualmente (FRENKEL y GHIOTTO, 2019). Esto, a su vez, generaría un efecto en cadena: la competitividad asimétrica producto de la apertura indiscriminada y la ausencia de protecciones por parte del bloque darían como resultado mayores presiones para “adaptar" los entramados productivos de los países sudamericanos. Por mencionar un caso, el

10 Aunque Fernández expresó su desacuerdo en promover medidas de flexibilización laboral similares a las aplicadas en Brasil, sí llegó a manifestarse a favor de abrir las negociaciones de los convenios colectivos de trabajo y actualizarlos "a la economía moderna" (BURGUEÑO, 2019).

Barbarói, Santa Cruz do Sul, n. 57, p.<o8-28>,jul./dez. 2020 
empresario agropecuario argentino Gustavo Grobocopatel manifestó que el tratado "obliga" al sector privado y al público a realizar cambios para lograr mayor competitividad y en base a ello agregó que "hay que dejar que algunos sectores de la economía desaparezcan" (LA NACIÓN, 2019).

Pero el panorama se vuelve aún más complicado si se tienen en cuenta los cambios en el escenario internacional. La visión globalization friendly enarbolada por algunos gobiernos liberalconservadores de la región tuvo que enfrentar una situación global adversa a sus aspiraciones. Por caso, la orientación aperturista del Mercosur chocaría con la oleada proteccionista impulsada por el gobierno de Donald Trump en Estados Unidos. La restricción a la importación de limones de origen argentino, a la carne bovina brasileña, sumada a la decisión de aumentar los aranceles a la entrada biocombustibles y al intento -posteriormente desactivado- de imponer aranceles a la importación de acero y aluminio son indicadores que revelan el desfasaje entre las políticas de apertura económica y las tendencias comerciales a nivel global. A esto hay que agregar un dato no menor: China, quien se ha transformado en el principal socio comercial de los países del Mercosur, propicia negociaciones comerciales bilaterales y simultáneas, conformando un esquema radial de vinculación. Esto no hace más que debilitar la posición relativa en las negociaciones y profundizar la asimetría entre el gigante asiático y los países de la región.

\section{Conclusiones}

Los puntos presentados anteriormente indican que el Mercosur -y la integración latinoamericana- se encuentra en un momento de repliegue y reconfiguración. Si bien dicho proceso se inició cuando aún gobernaban las administraciones neodesarrollistas, la llegada de gobiernos neoliberales ha profundizado las tendencias aperturistas y flexibilizadoras en el plano económico y la polarización en el ámbito político-ideológico. Las proclamas para "hacer converger" el Mercosur con la Alianza del Pacífico son un claro indicador de lo primero. La exclusión de Venezuela y la decisión de Argentina, Brasil, Chile, Colombia, Paraguay y Perú de retirarse de la UNASUR, son ejemplos de lo segundo. En este marco, las visiones de un regionalismo en clave autonómica, multidimensional e inclusiva han sido desechadas como opciones estratégicas de desarrollo.

En un mismo sentido, las reformas en el mercado del trabajo se han transformado en un tema con cada vez más protagonismo en las discusiones nacionales y regionales. Reducir el costo 
laboral es una necesidad imperiosa para aquellas estrategias económicas que tienen como horizonte la atracción inversiones externas y la inserción "competitiva" en las cadenas globales de valor. Sin embargo, vale señalar, pensando en el presente y el futuro, una lección del pasado: las reformas de este tipo aplicadas en años anteriores resultaron ser insuficientes para atraer capitales foráneos y terminaron afectando negativamente la situación de los trabajadores donde se implementaron (BOHOVSLAVSKY, 2017).

Vale aclarar también que si bien esta no es la primera oleada de reformas laborales en los países del Mercosur, el carácter individual y nacional con el que se han llevado a cabo es un indicador de las falencias del bloque para elaborar políticas públicas regionales. En la práctica, más que aunar posiciones para "mejorar la competitividad de la región", los gobiernos terminaron compitiendo entre sí para atraer inversiones, contradiciendo en algunos casos lo acordado previamente. Esto fue apuntado por el gobierno uruguayo de Tabaré Vázquez luego de que se aprobara la reforma laboral en Brasil, al cuestionar la contradicción entre los cambios en la jornada de trabajo que habilita esa reforma y lo establecido en la Declaración Sociolaboral del Mercosur (Diario Río Negro, 2017). El gobierno oriental incluso llegaría a manifestar la necesidad de tratar en el seno del Mercosur los efectos de la nueva legislación brasileña, abriendo un conflicto entre ambos países.

En tercer lugar, las tendencias comerciales proteccionistas en el ámbito global indican que el escenario político y económico dista largamente de ser una reproducción simple del esquema de globalización neoliberal que dio lugar a la creación del Mercosur en los años noventa. Las críticas al llamado "orden liberal mundial" por parte del país que fuera su principal impulsor representa un obstáculo a la estrategia aperturista de los gobiernos neoliberales sudamericanos. Asimismo, los vaivenes sobre el acuerdo con la Unión Europea -símbolo del renovado perfil que se pretende para el bloque conosureño- ocurren en un momento gran incertidumbre. Especialmente, a partir de la salida del Reino Unido del esquema comunitario y las crecientes manifestaciones en otros países europeos contrarias a ratificar el tratado.

De igual forma, orientar los esfuerzos hacia China y algunos países asiáticos - quienes mejor enarbolan, hoy, las banderas de la globalización- tampoco ofrece un panorama demasiado alentador, en especial si se enfoca la agenda laboral. Si bien es cierto que el comercio con esa zona del planeta se está tornando cada vez más relevante y que el constante crecimiento de las economías asiáticas ofrece nuevos nichos para la colocación de productos, la naturaleza de estos vínculos, así Barbarói, Santa Cruz do Sul, n. 57, p.<o8-28>, jul./dez. 2020 
como están planteados, no deja de ser marcadamente asimétrica. Por un lado, expone las dificultades de las industrias regionales para competir con economías que cuentan con un fuerte respaldo estatal y con altos niveles de flexibilización laboral. Por otro lado, se multiplican los incentivos para seguir profundizando la primarización de las economías del Mercosur.

En definitiva, plantear una vinculación acrítica tanto con aquellos que hoy cuestionan la globalización neoliberal como con quienes se presentan como sus más fervores defensores nos lleva al mismo callejón sin salida: abrir más las economías y flexibilizar todavía más los mercados de trabajo como el único camino posible. Resta, entonces, plantear una alternativa de cara al futuro. Cualquiera sea el prisma ideológico del que se parta, en tanto la integración sea entendida como un mero vehículo para fortalecer las economías nacionales -aun cuando ello afecte la consolidación de un mercado regional- resultará imposible trascender los vicios y las vulnerabilidades estructurales. En este escenario se torna imperioso crear mecanismos que articulen políticas comunes y transformen las voluntades nacionales en políticas públicas regionales, si lo que se quiere es construir un entramado que mitigue las pulsiones cortoplacistas y potencie la capacidad de negociación de la región. Este entramado, además, debe incluir una activa participación social más vinculante, más inclusiva y menos declarativa.

\title{
NEOLIBERAL RECONSTITUTION AND LABOUR MARKET REFORMS IN MERCOSUR: THE CASES OF ARGENTINA AND BRAZIL (2015-2020)
}

\begin{abstract}
The article analyzes the changes in the South Common Market (Mercosur) labour agenda and its relation with the arrival of neoliberal governments in Argentina and Brazil neoliberal governments in the mid-2010s. In this regard, the article pays particular attention to the labour market reform initiatives promoted by the governments of Michel Temer and Jair Bolsonaro in Brazil and Mauricio Macri in Argentina. Reforms that sought promoting the "competitiveness" of the bloc's economies, bolstering a peripheric integration into global value chains.

Key Words: Mercosur; neoliberalism; labour market agenda; Argentina; Brasil.

\section{RECONSTITUIÇÃO NEOLIBERAL E REFORMAS DO MERCADO DE TRABALHO NO MERCOSUL: OS CASOS DA ARGENTINA E DO BRASIL (2015-2020)}

\section{Resumo}

Este trabalho analisa mudanças na agenda trabalhista dos países do Mercosul e sua vinculação ao perfil assumido pelo bloco após a chegada de governos neoliberais em meados da década de 2010. Nesse contexto, é dada especial atenção às iniciativas de reforma do mercado de trabalho promovidas pelos governos de Michel Temer e Jair Bolsonaro no Brasil e Mauricio Macri na 
Argentina. Reformas que buscavam promover a "competitividade" das economias do bloco, apostando na integração periférica nas cadeias globais de valor.

Palavras-Chave: Mercosul; neoliberalismo; agenda trabalhista; Argentina; Brasil.

\section{REFERENCIAS}

ACEMOGLU, D. Y RESTREPO, P. Robots and Jobs: Evidence from US Labor Markets.

Journal of Political Economy, n.0, 2019.

BONELLI, M. Macri y una reforma laboral que es más fuerte de lo esperado. Clarín, Buenos Aires, 3 de noviembre de 2017. Disponible en https://www.clarin.com/opinion/macri-reformalaboral-fuerte-esperado_0_BkeILXF0-.html Acceso el: 3 de abril de 2020.

BAE NEGOCIOS. Guedes amenazó con restricción de garantías constitucionales en Brasil. 26 de noviembre de 2019. Disponible en: https://www.baenegocios.com/mundo/Guedesamenazo-con-restriccion-de-garantias-constitucionales-en-Brasil-20191126-0016.html Acceso el: 4 de abril de 2020.

BARRAZA, J. A. Flexibilización y Precarización laboral en la industria automotriz cordobesa. Villa María: Universidad Nacional de Villa María, 2017.

BERRINGER, T. Y FORLINI, L. Crise política e mudança na política externa no governo Temer: contradições no seio da burguesia interna brasileira. Conjuntura Austral, vol. 9, no. 48, p. 5-19. 2018.

BIZZOZERO, L. América Latina a inicios de la segunda década del siglo XXI: entre el regionalismo estratégico y la regionalización fragmentada. Revista Brasileira de Política Internacional, vol. 54, no. 1, p. 29-43. 2011.

BOTTO, M. La Integración Regional en América Latina: Quo Vadis? El Mercosur desde una perspectiva sectorial y comparada. Buenos Aires: Eudeba, 2015.

BRESSER- PEREIRA, L. C.. Estado y mercado en el nuevo desarrollismo. Nueva Sociedad, n.210, p. 110-125, 2007.

Reflexões sobre o Novo Desenvolvimentismo e o Desenvolvimentismo Clássico.

Revista de Economia Política, v. 36 n. 2, 143, 2016.

BRESSER-PEREIRA, L. C., OREIRO, J. L. y MARCONI, N.. Developmental

Macroeconomics: new developmentalism as a growth strategy. New York: Routledge, 2014.

¿Qué nos enseñan los 20 años del Mercosur? Nueva Sociedad, n. 32, p. 17-25, 2011.

BRICEÑO RUIZ, J. Ejes y modelos en la etapa actual de la integración económica regional en América Latina. Estudios internacionales (Santiago), v. 45 n.175, p. 9-39, 2013. 
El regionalismo estratégico en las interacciones entre Estados Unidos y Brasil en el ALCA: un análisis desde el liberalismo intergubernamental. En: P. DE LOMBAERDE y J. BRICEÑO-RUIZ (eds.), Del regionalismo latinoamericano a la integración interregional. Madrid: Siglo XXI - Fundación Carolina, p. 99-133, 2008.

BURGUEÑO, C. Alberto Fernández derribará un mito: abrirá discusiones de los convenios colectivos. Buenos Aires, Ámbito Financiero, 15 de noviembre 2019. Disponible en: https://www.ambito.com/economia/cgt/alberto-fernandez-derribara-un-mito-abrira-discusioneslos-convenios-colectivos-n5065844 Acceso el 10 de abril de 2020.

CABALLERO, S. Reformas para el Mercosur: ¿Solución o nuevos problemas? Análisis Carolina, no. 5, 2019.

CAETANO, G. Parlamento Regional y Sociedad Civil en el Proceso de Integración ¿Una nueva oportunidad para «otro» Mercosur? Friedrich Ebert Stiftung. Montevideo, Uruguay, 2006.

. (Ed). Mercosur 20 años. Montevideo: CEFIR, 2010.

CARRAU, N. La Coordinadora de Centrales Sindicales del Cono Sur. Un actor con mirada regional en el MERCOSUR. Montevideo: Friedrich Ebert Stiftung, 2008.

CESIT. Dossie Reforma Trabalhista. Unicamp: Instituto de Economía, 2017. Disponible en http://www.cesit.net.br/wp-content/uploads/2017/06/Dossie-14set2017.pdf Acceso el: 2 de abril de 2020.

COMINI, N., y FRENKEL, A. La política internacional de América Latina: más atomización que convergencia. Nueva Sociedad, n. 271, p. 117-129, 2017.

COOK, M. L. Unions and Labor Policy Under Left Governments in Latin America. Revista Trabajo, v. 5 n. 7, p. 55-73, 2011.

(2007) The politics of labor reform in Latin America : between flexibility and rights. University Park: The Pennsylvania State University Press.

DIARIO RÍO NEGRO. Uruguay, preocupado por la reforma laboral de Brasil, Viedma, 15 de Agosto de 2017. Disponible en http://www.rionegro.com.ar/mundo/uruguay-preocupado-porla-reforma-laboral-de-brasil-BA3354021. Acceso el: 4 de abril de 2020.

DOBRUSIN, B. Argentina's labour movement and trade policy: the case of CTA within Mercosur during the Kirchner's government. Political perspectives, vol. 6, no. 2, p. 58-77. 2012.

FÉLIZ, M. Neo-developmentalism: Beyond neoliberalism? Capitalist crisis and Argentina's development since the 1990s. Historical Materialism, v. 20 n. 2, p. 105-123, 2012. 
FERNÁNDEZ, A. A. Sobre política comparada: Reformulación de una tipología de los estados actuales. En A. A. Fernández (Ed.), Estudios de política comparada: perspectivas, experiencias y debates (pp. 15-62). Rosario: Universidad Nacional de Rosario, 2004.

FRENKEL, A. Y GHIOTTO, L. Los perdedores de siempre. Apuntes sobre el acuerdo entre la Unión Europea y el Mercosur. Nueva Sociedad, edición digital, julio de 2019. Disponible en: https://nuso.org/articulo/ue-mercosur-europa-ganadores-perdedores-integracion/ Acceso el 12 de abril de 2020.

FREY, C. Y OSBORNE, M. The future of employment: How susceptible are jobs to computerisation? Technological forecasting and social change, vol. 114, p. 254-280. 2017.

GRAETZ, G. Y MICHAELS, G. Robots at work. Review of Economics and Statistics, vol. 100, no. 5, p. 753-768. 2018.

HERRERA, J. C. Prosur, el nuevo mecanismo para no integrar a Latinoamérica. The New York Times, 9 de abril de 2019. Disponible en: https://www.nytimes.com/es/2019/04/09/espanol/opinion/prosur-unasur-america-latina.html Acceso el 4 de abril de 2020.

INCHAUSPE, E., y PEROTTA, D. EI MERCOSUR ¿cambio o continuidad? En IV Congreso de Relaciones Internacionales, Universidad Nacional de La Plata, 2008.

IRAJÁ, V. A reforma silenciosa de Paulo Guedes para revolucionar a máquina federal. Revista Veja, 13 de diciembre de 2019. Disponible en https://veja.abril.com.br/economia/areforma-silenciosa-de-paulo-guedes-para-revolucionar-a-maquina-federal/ Acceso el 20 de junio de 2020.

LA NACIÓN. Gustavo Grobocopatel: "Hay que dejar que algunos sectores desaparezcan", Buenos Aires, 1 de julio de 2019. Disponible en:

https://www.lanacion.com.ar/economia/grobocopatel-hay-que-dejar-que-algunos-sectoresdesaparezcan-nid2263267 Acceso el 20 de junio de 2020.

LARIVIÈRE, B. et. al. Service Encounter 2.0: An investigation into the roles of technology, employees and customers. Journal of Business Research, vol. 79, p. 238-246. 2017.

MAKRIDAKIS, S. The forthcoming Artificial Intelligence (AI) revolution: Its impact on society and firms. Futures, vol. 90, p. 46-60. 2017.

MALAMUD, A. Presidential diplomacy and the institutional underpinnings of Mercosur: An Empirical Examination, Latin American Research Review, v. 40 n. 1, p. 138-164, 2005.

MERCADO COMÚN DEL SUR. Declaración Sociolaboral del Mercosur, 1998. Disponible en http://www.mercosur.int/innovaportal/file/4506/1/cmc_1998_ata02_declaracion_es_sociolaboral. pdf Acceso el 4 de abril de 2020. 
Declaración Sociolaboral del Mercosur-2015. Brasilia, 2015. Disponible en

http://www.cartillaciudadania.mercosur.int/uploads/Declaracion\%20Sociolaboral\%202015.pdf Acceso el 4 de abril de 2020.

MOREIRA, D. S. O. As Semelhanças Entre os Governos Temer e Bolsonaro na Política Externa (2016-2019). Revista Neiba, Cadernos Argentina Brasil, vol. 9, no. 1. 2020.

PAGNI, C. Bajar costos, la nueva obsesión de Macri. Buenos Aires, La Nación, 9 de enero de 2017. Disponible en https://www.lanacion.com.ar/1974167-bajar-costos-la-nueva-obsesion-demacri Acceso el 4 de abril de 2020.

PASSARINHO, N. 6 pontos da reforma econômica de Paulo Guedes que podem te afetar diretamente. BBC News Brasil, 6 de noviembre de 2019. Disponible en https://www.bbc.com/portuguese/brasil-50319850 Acceso el 20 de junio de 2020.

PERROTTA, D., y VAZQUEZ, M. EI MERCOSUR de las políticas públicas regionales. Las agendas en desarrollo social y educación. Montevideo: CEFIR - In Went, 2010.

QUILICONI, C. La encrucijada del regionalismo latinoamericano ¿Cambio de ciclo o retorno al regionalismo abierto? Línea Sur, Revista de Política Exterior, vol. 3, no. 14, p. 146157. 2018.

RIGGIROZZI, P., \& GRUGEL, J. Regional governance and legitimacy in South America: the meaning of UNASUR. International Affairs, v. 91 n. 4, p. 781-797, 2015.

RIGGIROZZI, P., y TUSSIE, D.. The rise of post-hegemonic regionalism: The case of Latin America. Dordrecht: Springer, 2012.

ROBLES, J. Integración regional, democracia y relaciones laborales contractuales: un análisis desde la experiencia sudamericana. Cuaderno de Relaciones Laborales: v.24, n.1, p. 57-83, 2006.

Balance y perspectivas de los organismos sociolaborales del Mercosur. Taller de Formación y Debate "El futuro del MERCOSUR", Buenos Aires, Fundación Friedrich Ebert, 2002. Disponible en: https://library.fes.de/pdf-files/bueros/argentinien/01924.pdf Acceso el 5 de abril de 2020.

ROJAS ARAVENA, F. Introducción. Cambios y nuevas tendencias en el sistema internacional: abriendo nuevos espacios de concertación e integración efectiva. En F. Rojas Aravena (Ed.), América Latina y el Caribe: Relaciones Internacionales en el siglo XXI.

Diplomacia de Cumbres y espacios de concertación regional y global (p. 412). Buenos Aires: Teseo, 2012.

SANAHUJA, J. A. Del regionalismo abierto al regionalismo post-liberal. Crisis y cambio en la integración regional en América Latina. Anuario de la integración regional de América Latina y el Gran Caribe, n. 7, p. 12-54, 2009. 
SANAHUJA, J. A. Y RODRÍGUEZ, J. D. Veinte años de negociaciones Unión EuropeaMercosur: Del interregionalismo a la crisis de la globalización. Documentos de Trabajo No13. Madrid: Fundación Carolina, 2019.

SCHWAB, K. La cuarta revolución industrial. Buenos Aires: Debate. 2016.

SERBIN, A. Regionalismo y soberanía nacional en América Latina: lecciones aprendidas y nuevos desafíos. Anuario Ceipaz, n. 4, p. 137-178, 2011.

SOTO, D. A. Technology and the future of work in emerging economies: What is different. OECD Social, Employment and Migration Working Papers, no. 236. 2020.

TEIXEIRA, M. O desmonte trabalhista e previdenciário: reinventando novas formas de desigualdades entre os sexos. Analise N. 26, FES-Brasil, 2017.

ZELICOVICH, J. Claves y tensiones de la estrategia argentina de política comercial externa en la búsqueda de una "inserción inteligente al mundo" (2015-2018). Latin American Journal of Trade Policy, no. 2, p. 49-66. 2018.

VAN EVERA, S. Guide to methods for students of political science. Cornell: Cornell University Press, 1997.

VÁZQUEZ, M. El Mercosur social. Cambio político y nueva identidad para el proceso de integración regional en América del Sur. En G. Caetano (Ed.), Mercosur 20 años. Montevideo: Centro de Formación para la Integración Regional - CEFIR, p. 165-185, 2011.

ZULETA. I. El plan secreto para bajar el costo laboral. Clarín, 2 de abril de 2017. Disponible en https://www.clarin.com/economia/plan-secreto-bajar-costo-laboral_0_Hy1KgN3ne.html Acceso el 4 de abril de 2020.

Data de recebimento: $25 / 04 / 2020$

Data de aceite: 17/08/2020

Sobre os autores:

Alejandro Frenkel é licenciado en Ciencia Política y Doctor en Ciencias Sociales, Universidad de Buenos Aires. Profesor adjunto de la Escuela de Política y Gobierno, Universidad Nacional de San Martín. Becario post-doctoral del Consejo Nacional de Investigaciones Científicas y Técnicas. Endereço Eletrônico: afrenkel@unsam.edu.ar

Bruno Dobrusin é graduado en International Development Studies and Politics, Trent University. Doctor en Ciencias Sociales, Universidad de Buenos Aires.. Endereço Eletrônico: bdobrusin@gmail.com 